\title{
Magnetar-driven explosions as power source of Gamma-ray Bursts and Supernovae
}

\author{
Felipe Olivares E. ${ }^{1,2}$ and Jochen Greiner ${ }^{3}$ \\ ${ }^{1}$ Departamento de Ciencias Fisicas, Universidad Andres Bello, Avda. Republica 252, Santiago, Chile \\ ${ }^{2}$ Millennium Institute of Astrophysics, Chile, email: f.olivares.e@gmail.com \\ ${ }^{3}$ Max-Planck-Institut für Extraterrestrische Physik, Giessenbachstr. 1, 85748 Garching, Germany
}

\begin{abstract}
We present photometry and spectroscopy of SN 2011kl associated to GRB 111209A. Peculiarities arise from both the ultra-long GRB and the very luminous SN, brighter than any other GRB-associated SN observed to date. We present unequivocal evidence in favor of a scenario where a newly-formed magnetar powers both the GRB and the SN.
\end{abstract}

Keywords. gamma rays: bursts, supernovae: individual (SN 2011kl)

Long-duration gamma-ray bursts (GRBs) are linked to massive stars stripped of their $\mathrm{H}$ and $\mathrm{He}$ envelopes through broad-lined supernovae (SNe) Ic. The first GRB-SN identified as magnetar-powered was SN 2006aj (XRF 060218), although evidence was not conclusive. The magnetar scenario is usually invoked for H-poor SLSNe, although evidence has suggested that GRB-SNe are also powered by magnetars, because (1) the SN drives the explosion in terms of energy and (2) the SN kinetic energy is strikingly similar to the rotational energy of a milisecond magnetar $\left(\sim 2 \times 10^{52} \mathrm{erg}\right)$.

The very luminous SN 2011kl was associated with the ultra-long GRB 111209A $\left(T_{90} \approx 4 \mathrm{hrs}\right)$ at $z=0.677$. Binary-channel formation of a low-metallicity BSG progenitor and tidal disruption were proposed as possible scenarios. However, the obvious SN bump in the photometric data, which consists of GROND grizJ bands and Swift/UVOT $u$ band (see Greiner et al. (2015) for multicolor light curves), discards a tidal disruption event. The luminosity of SN 2011kl peaks at 14 days after the explosion with $L_{\mathrm{bol}}=2.8 \times 10^{43} \mathrm{erg} \mathrm{s}^{-1}\left(M_{\mathrm{bol}}=20.0 \mathrm{mag}\right)$, which is three times brighter than previous GRB-SNe (Fig. 1, left panel). While the ${ }^{56} \mathrm{Ni}$-powered model of the pseudo-bolometric light curve delivers $M_{\mathrm{Ni}}=1.0 \pm 0.1 M_{\odot}$ and $M_{\mathrm{ej}}=3 \pm 1 M_{\odot}$, the magnetar model results in $P_{i} \approx 12 \mathrm{~ms}, B=(6-9) \times 10^{14} \mathrm{G}, M_{\mathrm{ej}}=2-3 M_{\odot}$ and $E_{\mathrm{k}}=(2-9) \times 10^{51} \mathrm{erg}$. The spectrum at around maximum looks almost featureless (Fig. 1, right panel). The low opacity at $300-400 \mathrm{~nm}$ is not compatible with $M_{\mathrm{Ni}} \approx 1 M_{\odot}$ and there is no evidence of freshly synthesized material. The estimated expansion velocity corresponds to $v_{\mathrm{ph}} \approx 21,000 \mathrm{~km} \mathrm{~s}^{-1}$, which is faster than super-luminous SNe (SLSNe; $\sim 10,000 \mathrm{~km} \mathrm{~s}^{-1}$ ). A BSG progenitor would have implied $\mathrm{H}$ lines in the spectrum and a slower light curve. A high luminosity due to CSM interaction would have implied strong Balmer lines. The correction for GRB-jet collimation has to be $<1 / 50$ to be compatible with the maximum magnetar energy, which is well within typical values for GRBs. Given that there is no evidence in the spectrum for such large ${ }^{56} \mathrm{Ni}$ mass and the blue spectrum of SN $2011 \mathrm{kl}$ resembles those of magnetar-powered SLSNe, we invoke the magnetar model for this association. This scenario offers a link between GRB-associated SNe, ultra-long GRBs, and SLSNe. 

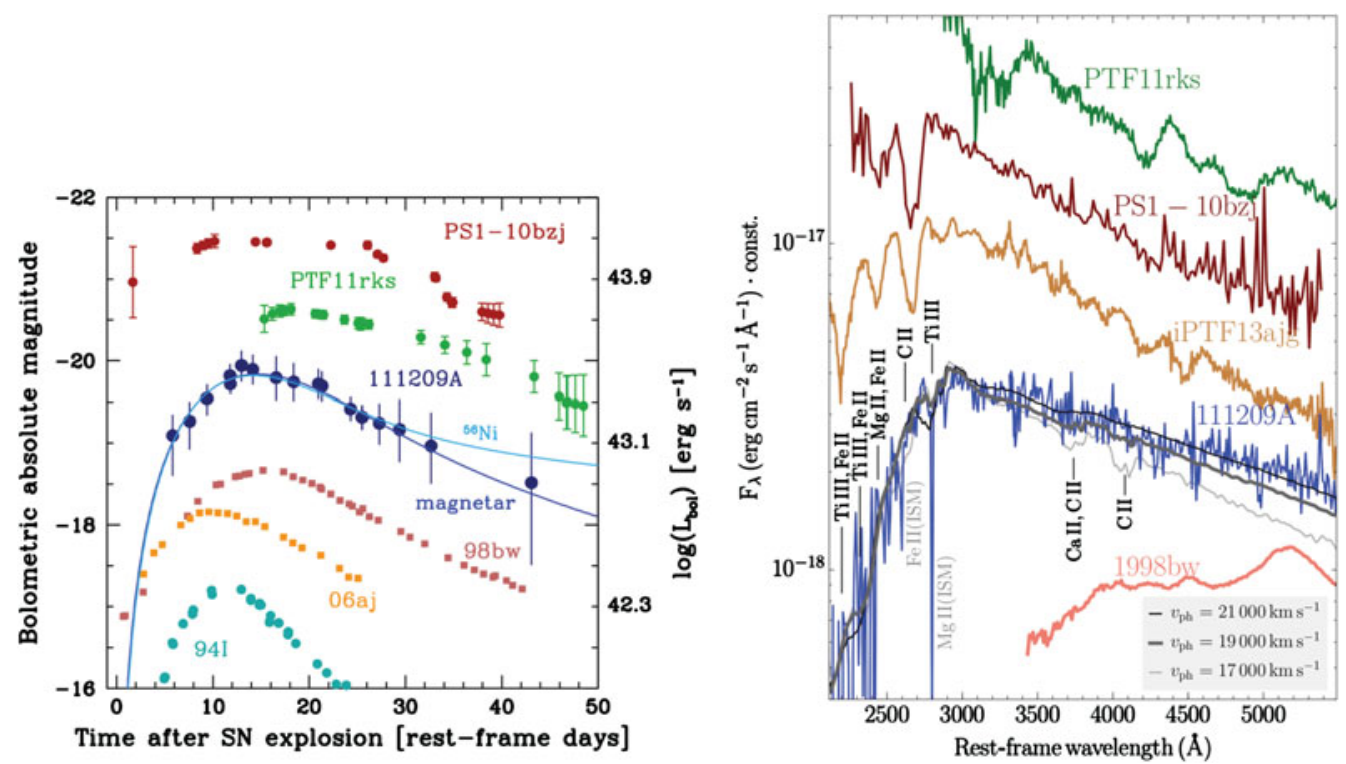

Figure 1. Bolometric light curve (left) and spectrum (right) of SN 2011kl compared to SLSNe and GRB-SNe. Refer to Greiner et al. (2015) for more details.

\section{References}

Greiner, J., Mazzali, P. A., Kann, D. A., Krühler, T., Pian, E., Prentice, S., Olivares, E., F., et al., 2015, Nature, 523, 189; and references therein. 